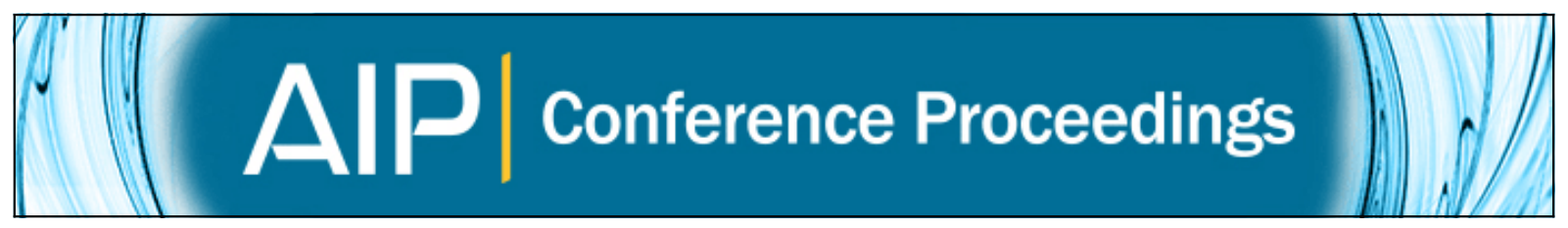

Polynomial Invariants for General Higher Spin Dirac Operators: A Toy Model

David Eelbode and Dalibor Šmíd

Citation: AIP Conference Proceedings 1281, 1504 (2010); doi: 10.1063/1.3498060

View online: http://dx.doi.org/10.1063/1.3498060

View Table of Contents: http://scitation.aip.org/content/aip/proceeding/aipcp/1281?ver=pdfcov

Published by the AIP Publishing

Articles you may be interested in

On an Inductive Construction of Higher Spin Dirac Operators

AIP Conf. Proc. 1281, 1500 (2010); 10.1063/1.3498058

Fischer Decompositions of Kernels of Hermitean Dirac Operators

AIP Conf. Proc. 1281, 1484 (2010); 10.1063/1.3498050

Explicit Formulae for Monogenic Projections

AIP Conf. Proc. 1048, 697 (2008); 10.1063/1.2991024

Symplectic Dirac Operator and its Higher Spin Analogues

AIP Conf. Proc. 1048, 674 (2008); 10.1063/1.2991017

The Generalised Dolbeault Complex for Four Dirac Operators in the Stable Rank

AIP Conf. Proc. 1048, 670 (2008); 10.1063/1.2991016 


\title{
Polynomial Invariants for General Higher Spin Dirac Operators: A Toy Model
}

\author{
David Eelbode* and Dalibor Šmíd ${ }^{\dagger}$ \\ ${ }^{*}$ Department of Mathematics and Computer Science, University of Antwerp, Campus Middelheim, G-Building, \\ Middelheimlaan 1, 2020 Antwerpen, Belgium \\ ${ }^{\dagger}$ Mathematical Institute, Charles University, Sokolovská 83, 186 75, Praha 8, Czech Republic
}

\begin{abstract}
We extend our previous results on spaces of polynomial invariants for Rarita-Schwinger operators acting on representations $\mathbb{S}_{k}$ with highest weight $\left(k+\frac{1}{2}, \frac{1}{2}, \ldots, \frac{1}{2}\right)$ to a more general setting. This setting may be seen as a toy model for the study of Higher Spin Dirac operators on general representations with half-integral highest weights, and already hints at an essential difference of the structure of the space of invariant differential operators on $\mathbb{S}_{k}$ and on a general representation.
\end{abstract}

Keywords: Clifford algebras, Dirac operators, representation theory

PACS: $02.20 . \mathrm{Qs}, 02.30 . \mathrm{Fn}$

\section{INTRODUCTION}

Clifford analysis is a generalization of complex analysis to a higher-dimensional setting. In the classical sense it studies behaviour of functions on a vector space with values in its Clifford algebra or the corresponding spinor representation. The cornerstone of the theory is the Dirac operator, which plays the role of the generalization of the Cauchy-Riemann operator from complex analysis. The standard references are for example [1,7]. Let us denote by $\mathbb{C}^{m}$ a vector space with a regular bilinear form $Q$, by $\left\{e_{1}, \ldots, e_{m}\right\}$ a basis orthonormal with respect to $Q$ and by $\mathbb{R}^{m}$ its real span. The Clifford algebra $\mathbb{C}_{m}$ is generated by relations $e_{i} e_{j}+e_{j} e_{i}=-2 \delta_{i j}$ and has a structure of a graded vector space $\mathbb{C}_{m}=\bigoplus_{k=0}^{m} \mathbb{C}_{m}^{(k)}$, where $\mathbb{C}_{m}^{(k)}$ is spanned by elements $e_{i_{1}} \ldots e_{i_{k}}, i_{1}<\ldots<i_{k}$. The space $\mathbb{C}_{m}^{(2)}$ with the commutator bracket is the Lie algebra $\mathfrak{s o}(m)$ of the group $\operatorname{Spin}(m)$ of all even Clifford product of unit vectors. The spinor representation $\mathbb{S}$ of $\operatorname{Spin}(m)$ arises by a standard construction using the Witt basis. For $m$ odd, $\mathbb{S}$ is irreducible with highest weight $\left(\frac{1}{2}, \ldots, \frac{1}{2}\right)$, for $m$ even it decomposes into two irreducible summands $\mathbb{S}^{+} \oplus \mathbb{S}^{-}$with weights $\left(\frac{1}{2}, \ldots \pm \frac{1}{2}\right)$. For the sake of convenience, we will restrict to the case of odd dimension from now on.

The Dirac operator $\underline{\partial}_{x}:=\sum_{i=1}^{m} e_{i} \partial_{x_{i}}$ acts on the space of functions $\mathscr{C}^{\infty}\left(\mathbb{R}^{m}, \mathbb{S}\right)$ as a $\operatorname{Spin}(m)$-invariant elliptic differential operator, which factorizes the Laplacian in $m$ dimensions: $\underline{\partial}_{x}^{2}=-\Delta_{m}$. The algebra of constant coefficient invariant differential operators on $\mathscr{C}^{\infty}\left(\mathbb{R}^{m}, \mathbb{S}\right)$ is generated by powers of the Dirac operator. Functions in the kernel of the Dirac operator are called monogenic and play a similar role for the theory as holomorphic functions in the classical complex analysis do.

In the last decade there has been an interest $[3,4,5,12]$ in extending the techniques of Clifford analysis to the so called higher spin representations. In a broad sense, the term higher spin representation refers to any irreducible representation $\mathbb{S}_{\lambda}$ of $\operatorname{Spin}(m)$ with half-integral highest weight $\lambda^{\prime}=\lambda+\left(\frac{1}{2}, \ldots, \frac{1}{2}\right)$. There is (up to normalization) a unique first order invariant differential operator

$$
\mathscr{R}_{\lambda}: \mathscr{C}^{\infty}\left(\mathbb{R}^{m}, \mathbb{S}_{\lambda}\right) \rightarrow \mathscr{C}^{\infty}\left(\mathbb{R}^{m}, \mathbb{S}_{\lambda}\right)
$$

given as the projection of the gradient of a function to the irreducible summand $\mathbb{S}_{\lambda}$ in $\left(\mathbb{R}^{m}\right)^{*} \otimes \mathbb{S}_{\lambda}$. For $\lambda=$ $(1,0, \ldots, 0)=:(1)$ the operator $\mathscr{R}_{(1)}$ is (the multidimensional analogue of) the Rarita-Schwinger operator. In [4] the generalized Rarita-Schwinger operators $\mathscr{R}_{k}$ acting on functions with values in $\mathbb{S}_{(k)}$ were studied and the structure of their kernels was described. In [9] we have shown that the space of all constant coefficient invariant differential operators on $\mathscr{C}^{\infty}\left(\mathbb{R}^{m}, \mathbb{S}_{(k)}\right)$ is generated by products of certain powers of the Laplacian and of the generalized RaritaSchwinger operators.

Recent efforts focus on extending these results to more general higher spin representations, with the so called Higher Spin Dirac operators acting upon them. It is convenient to realize the representation $\mathbb{S}_{(k)}$ as the space $\mathscr{M}_{k}(u)$

CP1281, ICNAAM, Numerical Analysis and Applied Mathematics, International Conference 2010, Vol. III edited by T. E. Simos, G. Psihoyios, and Ch. Tsitouras

(C) 2010 American Institute of Physics 978-0-7354-0836-4/10/\$30.00 
of all monogenic polynomials of order $k$ in an auxiliary variable $u$. Similarly, there can be introduced [6] spaces of two-variable polynomials satisfying certain first order differential equations serving as a model for the representation $\mathscr{S}_{(k, l)}$, where $(k, l):=(k, l, 0, \ldots, 0)$. The kernels for the two-variable case were completely described in [2] . The space

of all constant coefficient invariant differential operators for the special two-variable representation $\mathscr{S}_{(k, 1)}$ is studied here as a simple model for the general two-variable or even $p$-variable case.

\section{DIMENSION OF THE SPACE OF INVARIANTS}

The symbol of a constant coefficient invariant differential operator acting on $\mathscr{C}^{\infty}\left(\mathbb{R}^{m}, \mathbb{S}_{\lambda}\right)$ is an invariant element of $\mathscr{P}\left(\mathbb{R}^{m}\right.$, End $\left.\mathbb{S}_{\lambda}\right)$. If we abuse the notation a little and write instead of irreducible representations just their highest weights, we may decompose this space of polynomials into the sum

$$
\mathscr{P}\left(\mathbb{R}^{m}, \text { End } \mathbb{S}_{\lambda}\right)=\bigoplus_{i=0}^{\infty} \mathscr{P}^{i}\left(\mathbb{R}^{m}, \mathbb{C}\right) \otimes\left(\lambda^{\prime} \otimes \lambda^{\prime}\right)=\bigoplus_{i=0}^{\infty} \bigoplus_{j=0}^{\left\lfloor\frac{i}{2}\right\rfloor}(i-2 j) \otimes\left(\lambda^{\prime} \otimes \lambda^{\prime}\right)
$$

Invariants correspond to trivial summands in this space. A trivial summand in $(p) \otimes\left(\lambda^{\prime} \otimes \lambda^{\prime}\right)$ occurs precisely once for each summand $(p)$ in $\lambda^{\prime} \otimes \lambda^{\prime}$. Hence the problem of determining the dimension of the space of invariants reduces to decomposing the second tensor power of $\lambda^{\prime}$ modulo all summands not of the type $(p)$. There are several algorithms for tensor product decomposition, i.e. Brauer-Klimyk formula, Young tableaux, Littelman paths, Kashiwara crystal bases, but no general formula except for a few of very special cases. One such special formula due to [11] is

$$
\left(q_{1}\right) \otimes\left(q_{2}\right)=\bigoplus_{i=0}^{q_{2}} \bigoplus_{j=0}^{q_{2}-i}\left(q_{1}+q_{2}-i-2 j, i\right)
$$

for $q_{1} \geq q_{2} \geq 0$. In [9] we used this formula to obtain all summands of type $(p)$ in $\left(k_{1}\right)^{\prime} \otimes\left(k_{2}\right)^{\prime}, k_{1} \geq k_{2}$ :

$$
\left(k_{1}\right)^{\prime} \otimes\left(k_{2}\right)^{\prime}=\bigoplus_{i=0}^{2 k_{2}+1}\left(k_{1}-k_{2}+i\right) \oplus \mathbb{W}
$$

where $\mathbb{W}$ does not contain any summand of the type $(p)$. From this we have

Theorem 1 The space of invariant $p$-homogeneous polynomials with values in End $\mathbb{S}_{(k)}$ is $\left\lceil\frac{p+1}{2}\right\rceil$-dimensional for $p \leq 2 k$ and $k+1$-dimensional for $p \geq 2 k$.

A general formula for $(p)$-type summands in $\left(k_{1}, l_{1}\right)^{\prime} \otimes\left(k_{2}, l_{2}\right)^{\prime}$ is not yet available. However, for $\left(k_{1}, 1\right)^{\prime} \otimes\left(k_{2}, 1\right)^{\prime}$ we may use a generalization of formula 1 and proceed as in [9]. Using

$$
(1) \otimes\left(k_{1}\right) \otimes\left(k_{2}\right)=\left[\left(k_{1}+1\right) \otimes\left(k_{2}\right)\right] \oplus\left[\left(k_{1}-1\right) \otimes\left(k_{2}\right)\right] \oplus\left[\left(k_{1}, 1\right) \otimes\left(k_{2}\right)\right]
$$

and

$$
(1) \otimes\left(k_{1}, 1\right) \otimes\left(k_{2}\right)=\left[\left(k_{1}, 1\right) \otimes\left(k_{2}+1\right)\right] \oplus\left[\left(k_{1}, 1\right) \otimes\left(k_{2}-1\right)\right] \oplus\left[\left(k_{1}, 1\right) \otimes\left(k_{2}, 1\right)\right]
$$

we can express $\left(k_{1}, 1\right) \otimes\left(k_{2}, 1\right)$ in terms of several products of the type from formula 1 and their products with representations $(1)$ and $(1) \otimes(1)$. In a similar fashion as in [9] this allows to prove the formula

$$
\left(k_{1}, 1\right)^{\prime} \otimes\left(k_{2}, 1\right)^{\prime}=\left(k_{1}-k-2\right) \oplus\left(k_{1}-k_{2}+1\right) \oplus 2 \bigoplus_{i=1}^{2\left(k_{2}-1\right)}\left(k_{1}-k_{2}+1\right) \oplus\left(k_{1}+k_{2}\right)+\left(k_{1}+k_{2}+1\right) \oplus \mathbb{W}
$$

where $k_{1} \geq k_{2} \geq 1$ and $\mathbb{W}$ does not contain any summand of type $(p)$. It follows

Theorem 2: The space of invariant $p$-homogeneous polynomials with values in $\mathrm{End}_{(k, 1)}$ is 1-dimensional for $p=0,1$, $\left(2\left\lfloor\frac{p}{2}\right\rfloor+1\right)$-dimensional for $2 \leq p \leq 2 k$ and $2 k$-dimensional for $p \geq 2 k$. 


\section{BASIS OF THE SPACE OF INVARIANTS}

For the values in $\mathbb{S}_{k}$, two interesting sets that span the space of invariants were found in [9]. The simpler one (written in terms of the corresponding differential operators) is $\left\{\Delta^{i}\left(\mathscr{R}_{(k)}\right)^{j}, i \geq 0,2 k+1 \geq j \geq 0\right\}$. We see that as an algebra, the invariants are generated by the Laplacian and the generalized Rarita-Schwinger operator.

The other basis may look complicated at first glance, however, it gives much more insight into why the spaces of invariants have the dimension calculated before and how to generalize them to more general representations. If we modify the construction of the generalized Rarita-Schwinger operator $\mathscr{R}_{(k)}$ on $\mathbb{S}_{(k)}$ in that we project onto a summand $\mathbb{S}_{(k-1)}$ or $\mathbb{S}_{(k+1)}$ in $\left(\mathbb{R}^{m}\right)^{*} \otimes \mathbb{S}_{(k)}$, we get the so called twistor operators $\mathscr{T}_{(k-1),(k)}$ and $\mathscr{T}_{(k+1),(k)}$, respectively. If we denote

$$
\mathscr{G}_{l, k}:=\mathscr{T}_{(l),(l+1)} \mathscr{T}_{(l+1),(l+2)} \ldots \mathscr{T}_{(k-2),(k-1)} \mathscr{T}_{(k-1),(k)}
$$

and

$$
\mathscr{G}_{k, l}:=\mathscr{T}_{(k),(k-1)} \mathscr{T}_{(k-1),(k-2)} \ldots \mathscr{T}_{(l+2),(l+1)} \mathscr{T}_{(l+1),(l)}
$$

the compositions of twistor operators for $k>l$ (and set $\mathscr{G}_{k, k}:=1$ ), then the (even order) invariants on $\mathbb{S}_{(k)}$ are spanned by the operators $\Delta^{i} \mathscr{G}_{k, j} \mathscr{G}_{j, k}$ for $i \geq 0$ and $0 \leq j \leq k$. The operators $\mathscr{G}$ can be seen as non-recurring paths on a graph whose nodes are all weights of the type $(p)^{\prime}$ and an edge exists between $(p)^{\prime}$ and $(q)^{\prime}$ iff $|p-q|=1$. It is clear that invariant opeators of order $2 k$ corresponds to all paths from $(k)^{\prime}$ to $(j)^{\prime}$ with $k \geq j \geq 0$ and there is precisely $k+1$ of them, as expected from Theorem 1.

In the two-variable case we can introduce twistor operators in the same way, there will in general be four of them defined on $\mathbb{S}_{(k, l)}: \mathscr{T}_{(k-1, l),(k, l)}, \mathscr{T}_{(k+1, l),(k, l)}, \mathscr{T}_{(k, l-1),(k, l)}, \mathscr{T}_{(k, l+1),(k, l)}$, mapping to $\mathbb{S}_{(k-1, l)}, \mathbb{S}_{(k+1, l)}, \mathbb{S}_{(k, l-1)}, \mathbb{S}_{(k, l+1)}$, respectively. Again, the set of all weights $(k, l)^{\prime}$ forms nodes of a graph that has an edge between two nodes iff there is a twistor operator between the corresponding representations. For two weights $(k, l)^{\prime},(m, n)^{\prime}, k \geq m, l \geq n$ we may define the operators $\mathscr{G}_{(m, n),(k, l)}$ and $\mathscr{G}_{(k, l),(m, n)}$ as the composition of twistor operators along a path with minimal length connecting the two weights. It can be shown [10] that with a suitable choice of normalization, these operators do not depend on the choice of the path. Moreover $\mathscr{G}_{(m, n),(k, l)}$ and $\mathscr{G}_{(k, l),(m, n)}$ are zero for $m<l$. For a fixed $(k, l)^{\prime}$, the set of all weights $(m, n)^{\prime}, k \geq m, l \geq n, m \geq l$ is a $k-l+1$ by $l+1$ rectangle in the weight lattice. Let us call this rectangle the box of $(k, l)^{\prime}$, denoting it by the symbol $B(k, l)$. The set of operators

$$
I=\left\{\Delta^{i} \mathscr{G}_{(k, l),(m, n)} \mathscr{G}_{(m, n),(k, l)}, i \geq 0,(m, n)^{\prime} \in B(k, l)\right\}
$$

is a candidate for the basis of the space of even order invariants (Odd order invariants are elements of $I$ multiplied by $\left.\mathscr{R}_{(k, l)}\right)$. There are $(k-l+l)(l+1)$ elements of order $2 k$ in the set. According to Theorem 2, for $l=1$ the space of invariants in homogeneity $2 k$ has dimension $2 k \equiv(k-1+1)(1+1)$. The only thing that remains to be proved is thus the linear independence of the set $I$.

\section{PERSPECTIVES}

Computations show that the equality between the size of $B(k, l)$ and the dimension of the space of invariants (of a fixed, sufficiently high order) on $\mathscr{S}_{(k, l)}$ remains the same also for $l>1$. The box $B(k, l)$ also plays an essential role in the structure of the kernel of the Higher Spin Dirac operator $\mathscr{R}_{(k, l)}$, as shown in [2]. In fact, if $\lambda^{\prime}$ is a half-integral highest weight of a representation $\mathscr{S}_{\lambda}$, the notion of $B(\lambda)$ arises in a similar fashion as for the two-variable case, and there is strong computational evidence that the size of $B(\lambda)$ and the dimension of the space of invariants (of a fixed, sufficiently high order) on $\mathscr{S}_{\lambda}$ still agree. However, proofs either of the dimensionality of the space of invariants or of the linear independence of invariants or of the structure of the kernel get more and more technically difficult towards the general case. The chosen toy model with $\lambda=(k, 1)$ is important, because it is the simplest case, for which the box $B(\lambda)$ is non-trivial, i.e. does not contain every representation below $\lambda$ in Bruhat order.

\section{REFERENCES}

1. Brackx, F., Delanghe, R., Sommen, F., Clifford Analysis, Research Notes in Mathematics 76, Pitman, London, 1982.

2. Brackx, F., Eelbode, D., Van de Voorde, L., The polynomial null solutions of a higher spin Dirac operator in two vector variables, preprint 
3. Bureš, J., The Rarita-Schwinger operator and spherical monogenic forms, Complex Variables Theory Appl. 43 No. 1 (2000), pp. 77-108.

4. Bureš, J., Sommen, F., Souček, V., Van Lancker, P., Rarita-Schwinger type operators in Clifford analysis, Journal of Funct. Anal. 185 (2001), pp. 425-456.

5. Bureš, J., Sommen, F., Souček, V., Van Lancker, P., Symmetric analogues of Rarita-Schwinger equations, Annals of Global Analysis Geometry 21 No. 3 (2001), pp. 215-240.

6. Constales, D., Sommen, F., Van Lancker, P., Models for irreducible Spin (m)-modules.

7. Delanghe, R., Sommen, F., Souček, V., Clifford analysis and spinor valued functions, Kluwer Academic Publishers, Dordrecht, 1992.

8. Eelbode, D., Šmíd, D., Polynomial invariants for the Rarita-Schwinger operator, in Sabadini, I., Shapiro, M., Sommen, F. (eds.), Hypercomplex analysis, Birkhäuser, Basel (2009), pp. 125-135.

9. Eelbode, D., Šmíd, D., Algebra of invariants for the Rarita-Schwinger operators, Ann. Ac. Sci. Fennicae: Mathematica, 34, (2009), pp. 637-649.

10. Eelbode, D., , Šmíd, D., Factorization of Laplacians on Higher Spin Representations, in preparation.

11. Klimyk, A. U., Infinitesimal operators for representations of complex Lie groups and Clebsch-Gordan coefficients for compact groups, J. Phys. A: Math. Gen. 15 (1982), pp. 3009-3023

12. Van Lancker, P., Rarita-Schwinger fields in the half space, Complex Variables and Elliptic Equations 51 No. 5-6 (2006), pp. 563-579. 